\title{
Prevalence of permanent childhood hearing impairment in the United Kingdom and implications for universal neonatal hearing screening: questionnaire based ascertainment study
}

\author{
Heather M Fortnum, A Quentin Summerfield, David H Marshall, Adrian C Davis, John M Bamford
}

\begin{abstract}
Objective To estimate the prevalence of confirmed permanent childhood hearing impairment and its profile across age and degree of impairment in the United Kingdom.

Design Retrospective total ascertainment through sources in the health and education sectors by postal questionnaire.

Setting Hospital based otology and audiology departments, community health clinics, education services for hearing impaired children.

Participants Children born from 1980 to 1995, resident in United Kingdom in 1998, with severe permanent childhood hearing impairment (hearing level in the better ear $>40 \mathrm{~dB}$ averaged over $0.5,1,2$, and $4 \mathrm{kHz}$ ).

Main outcome measures Numbers of cases with date of birth and severity of impairment converted to prevalences for each annual birth cohort (cases/1000 live births) and adjusted for underascertainment. Results 26000 notifications ascertained 17160 individual children. Prevalence rose from 0.91 (95\% confidence interval 0.85 to 0.98 ) for 3 year olds to 1.65 (1.62 to 1.68 ) for children aged 9-16 years. Adjustment for underascertainment increased estimates to 1.07 (1.03 to 1.12) and 2.05 (2.02 to 2.08). Comparison with previous studies showed that prevalence increases with age, rather than declining with year of birth.

Conclusions Prevalence of confirmed permanent childhood hearing impairment increases until the age of 9 years to a level higher than previously estimated. Relative to current yields of universal neonatal hearing screening in the United Kingdom, which are close to $1 / 1000$ live births, $50-90 \%$ more children are diagnosed with permanent childhood hearing impairment by the age of 9 years. Paediatric audiology services must have the capacity to achieve early identification and confirmation of these additional cases.
\end{abstract}

\section{Introduction}

Permanent childhood hearing impairment can have a devastating impact on communication skills, ${ }^{1}$ educational attainment, ${ }^{2}$ and quality of life, ${ }^{34}$ with a high cost to society. ${ }^{5}$ Improved outcomes for children with congenital impairment are associated with confirmation and intervention by 6 months of age. ${ }^{6}$ Yet the median age of confirmation of congenital impairments has exceeded 18 months, even in regions of the United Kingdom and United States with good paediatric audiology services. ${ }^{78}$ Universal neonatal hearing screening $^{910}$ has the potential to reduce the age at confirmation of congenital impairments. ${ }^{11}$ However, not all hearing impairments manifest themselves at birth, and screening programmes must be complemented by services that can confirm and manage cases where impairment first shows itself postnatally. No national register of hearing impaired children exists for the United Kingdom, and accurate estimates of the prevalence of permanent childhood hearing impairment and of its profile across age and degree of impairment are unavailable. We have provided such estimates at a time when paediatric audiology services in the United Kingdom are being transformed by the introduction of universal neonatal hearing screening ${ }^{12}$ and the modernisation of hearing aid services. ${ }^{13}$

Previous studies of the prevalence of permanent childhood hearing impairment display two limitations. ${ }^{78-16}$ Firstly, they ascertained relatively small samples (under 700 children) and so did not define the relation between prevalence, age, and degree of impairment precisely. Secondly, they did not estimate the extent of underascertainment. We examined these issues by estimating prevalence from a total ascertainment of hearing impaired children in the United Kingdom $(>17000)$ and by employing capture-recapture analysis $^{1718}$ to adjust for underascertainment. We estimated the prevalence of confirmed cases of permanent hearing impairment, including congenital, late onset, and acquired cases. Capture-recapture can correct for underascertainment of confirmed cases. It does not discover cases that have not been confirmed.

\author{
MRC Institute of \\ Hearing Research, \\ University Park, \\ Nottingham \\ NG7 2RD \\ Heather M \\ Fortnum \\ epidemiologist \\ A Quentin \\ Summerfield \\ deputy director \\ David H Marshall \\ statistician \\ Adrian C Davis \\ epidemiologist \\ Human \\ Communication \\ and Deafness \\ Group, University \\ of Manchester, \\ Manchester \\ M13 9PL \\ John M Bamford \\ professor of audiology \\ and education of the \\ deaf \\ Correspondence to: \\ Dr Fortnum \\ hf@ihr.mrc.ac.uk
}

BMJ 2001;323:1-6 


\section{Glossary}

Congenital impairment-A hearing impairment that is recognised at birth or that is believed to have been present since birth

Progressive impairment $-\mathrm{A}$ hearing impairment that may manifest itself at birth or postnatally and that worsens over time

Late onset impairment-A hearing impairment that first manifests itself postnatally and cannot be attributed to an identifiable exogenous cause

Acquired impairment-A hearing impairment that first manifests itself postnatally and can be attributed to an identifiable exogenous cause

Confirmation of hearing impairment-The outcome of the process of establishing that a child is hearing impaired

Notification-The contribution by an informant of data describing a child who meets the inclusion criteria for the study

Ascertainment-The identification by the research team of an individual child from one or more notifications Total ascertainment-The process of attempting to ascertain all cases in a population

Prevalence of confirmed cases-The number of children per thousand live births in an annual birth cohort with confirmed permanent bilateral childhood hearing impairment

\section{Methods}

\section{Case definition}

Cases were children resident in the United Kingdom during 1998, born between 1 January 1980 and 31 December 1995 inclusive, with confirmed permanent bilateral hearing impairment exceeding $40 \mathrm{~dB} \mathrm{HL}$ (hearing level) (average of pure tone thresholds at 0.5, 1,2 , and $4 \mathrm{kHz}$ in the better hearing ear).

\section{Ascertainment}

Children were ascertained through professionals with responsibility for the provision of audiological health care $(n=473)$ and education $(n=434)$ to hearing impaired children. During 1998, professionals were asked by mail to complete a one page form for each case known to them. Minimal data were requested to maintain anonymity and maximise compliance: ini-

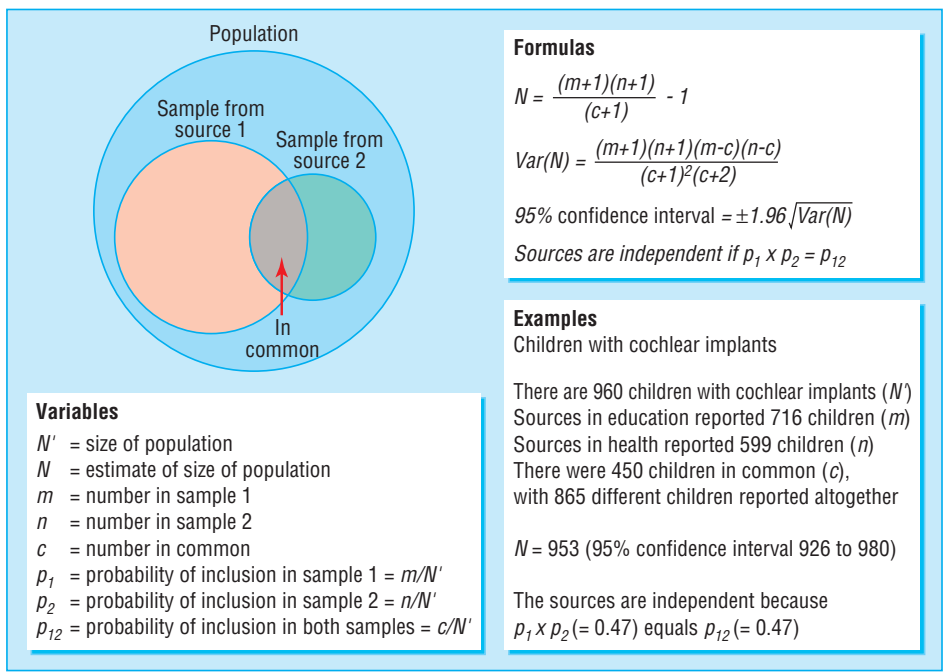

Fig 1 General methods of capture-recapture illustrated with data from validation exercise tials, sex, date of birth, home postcode, average hearing level in the better ear, presumed age at onset of hearing impairment, aetiology, other disabilities, parents' preferred language, and details of the source. We sent one reminder to non-responders after four months.

We combined lists of children notified by different informants. Duplicate entries were minimised by an interactive database that alerted us to possible matches by displaying details of children with similar demographic data. All data for such children were then compared to minimise the possibility of duplication of cases. We classified cases according to whether they were notified only by sources in the health sector, only by sources in the education sector, or by at least one source in each sector.

\section{Capture-recapture}

Capture-recapture techniques can estimate the size of populations from restricted samples (fig 1), and hence can estimate the extent of underascertainment in population surveys. Its use is valid when four requirements are met: the population does not change between compilation of the lists from different sources; cases from different sources can be matched; each member of the population has the same chance of inclusion in each list; and the sources are independent. The first three requirements were met by conducting the ascertainment over a short time span, by obtaining several identifiers for each case, and by using distinct sources with obligatory responsibilities for all hearing impaired children.

The last requirement is more demanding. ${ }^{19}{ }^{20}$ Independence can be shown if the probability of notification by both sources equals the product of the probabilities of notification by each source individually (fig 1). These quantities cannot be estimated from the data because each requires knowledge of the size of the population $\left(N^{\prime}\right)$. Separate evidence that the sources are independent is required. Accordingly, we counted all members of a special subset of the cases, comprising children with cochlear implants. They were reported additionally and independently of other sources in the health sector by the coordinators of the 16 clinical programmes that provide long term management to all children with implants in the United Kingdom. In this way $N$ 'for the subset was measured, which allowed us to test the independence of education sources from other sources in the health sector.

\section{Analysis}

We applied capture-recapture using conventional formulas to estimate the size of populations (fig 1). ${ }^{17}$ We used live birth statistics ${ }^{21}$ to convert counts of children into prevalence rates per 1000 live births. We also calculated confidence intervals for observed prevalences, assuming that counts obeyed Poisson probabilities, and for prevalences adjusted by capture-recapture using conventional formulas. ${ }^{17}$

\section{Results}

\section{Response rate}

Geographical coverage was comprehensive: professionals reported children from every postcode area in the United Kingdom, and only two of 122 postcode areas were not covered by professionals from both health and education. Of the 473 health professionals 


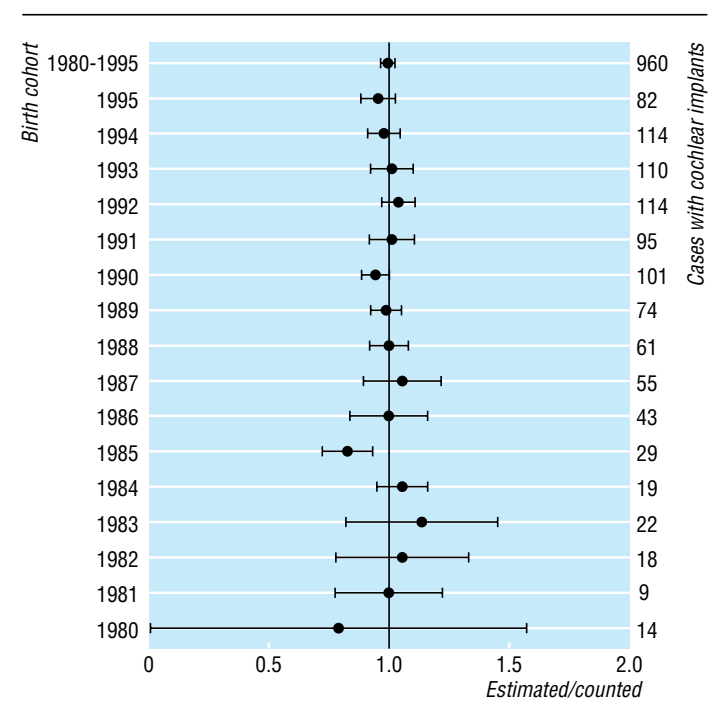

Fig 2 Ratios (and 95\% confidence intervals) of numbers of children with cochlear implants estimated by capture-recapture ("estimated") to number reported by paediatric cochlear implant programmes ("counted" and tabulated against right hand axis) for individual birth cohorts and 1980-95 cohorts combined

contacted, $122(26 \%)$ no longer had main responsibility for children in their geographical area. There was considerable overlap of institutional affiliation among the remainder, with the result that only 191 (54\%) individually provided data. Of the 434 education professionals contacted, 23 had retired. Of the remainder, 295 (72\%) provided data.

\section{Validation of capture-recapture}

The example in figure 1 documents the results of the validation exercise and confirms the independence of the sources. The 95\% confidence interval of the estimated number of children with implants embraces the true value overall and in 15 of 16 individual birth cohorts (fig 2). Application of capture-recapture to the remainder of the data was therefore justified.

\section{Prevalence}

We received over 26000 ascertainment forms. After we eliminated duplicate notifications the number ascertained was 17160 individual children: 11577 from health sources, 12107 from education sources, and 6524 from both. We calculated prevalence from observed counts and from counts adjusted by capturerecapture (fig 3). Prevalence declined for children in the oldest two cohorts (1981 and 1980), who were aged from 16 to 18 years when the ascertainment was conducted. Some would have left school and not been included in the education list; some would have transferred from paediatric to adult hearing services. Both effects violate the third requirement for capturerecapture to be valid. Hence these cohorts were excluded.

Using the observed data for all levels of severity combined, we compared prevalence in each cohort in turn with prevalence aggregated across all older cohorts. This procedure distinguished the 1982-9 cohorts as a group from the younger cohorts (Poisson probabilities, $\mathrm{P}<0.05$ ). Table 1 shows a rise in prevalence from age 3 to age 9 of $81 \%$ in observed values and $92 \%$ in adjusted values for impairments
$>40$ dB. A significant rise was also seen for each degree of impairment.

Two hypotheses might explain this result: either confirmed prevalence increases with age or prevalence has decreased with time. The current retrospective study did not follow cohorts over time, so to distinguish the hypotheses we compared current data with data obtained in studies conducted earlier, ${ }^{78}{ }^{14}$ aligning the data by birth cohort and separately by age. The most relevant earlier study was a total ascertainment of children with permanent hearing impairment in the Trent health region (population 4.7 million) in $1995 .^{7}$ This study reported an aggregate prevalence for the birth cohorts 1985-90 (age 5-10 years) of 1.33/1000 live births $(95 \%$ confidence interval 1.22 to 1.45$)$. In the present study, the observed aggregate prevalence in the same cohorts (age 8-13 years) was significantly higher at $1.63 / 1000$ (1.59 to 1.67 ), whereas prevalence among children matched for age (cohorts 1988-93) was not significantly different at $1.44 / 1000$ (1.41 to

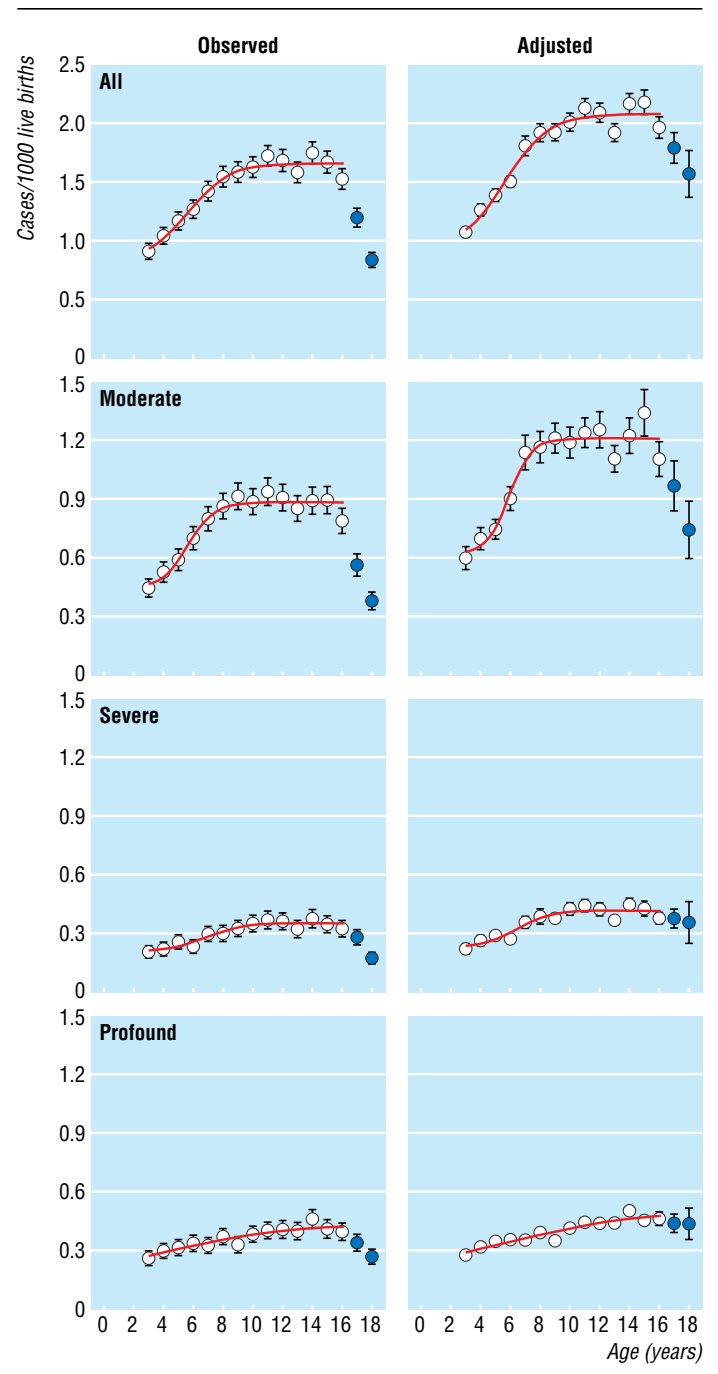

Fig 3 Profiles of observed and adjusted prevalence of permanent childhood hearing impairment with age and by degree of impairment (all: >40 dB HL; moderate: 41-70 dB HL; severe: 71-95 dB HL; profound: $>95 \mathrm{~dB} \mathrm{HL}$ ). ${ }^{22}$ Points plotted as filled circles were excluded from some analyses. 95\% confidence intervals included where they exceed width of plotting symbol. Continuous lines are best fitting sigmoidal functions 
Table 1 Observed and adjusted prevalences as cases per 1000 live births ( $95 \%$ confidence intervals) for single birth cohort with age of 3 years (1995) and for aggregate of birth cohorts with ages ranging from 9 to 16 years (1982-9)

\begin{tabular}{|c|c|c|c|c|}
\hline \multirow[b]{2}{*}{ Severity (dB HL) } & \multicolumn{2}{|c|}{ Observed prevalence } & \multicolumn{2}{|c|}{ Adjusted ${ }^{\star}$ prevalence } \\
\hline & 1995 & $1982-9$ & 1995 & $1982-89$ \\
\hline$>40$ & 0.91 (0.85 to 0.98$)$ & 1.65 (1.62 to 1.68$)$ & 1.07 (1.03 to 1.12$)$ & 2.05 (2.02 to 2.08) \\
\hline $41-70$ & $0.45(0.40$ to 0.50$)$ & $0.89(0.86$ to 0.91$)$ & 0.60 (0.54 to 0.66$)$ & 1.21 (1.18 to 1.24$)$ \\
\hline $71-95$ & $0.20(0.17$ to 0.24$)$ & 0.35 (0.33 to 0.36$)$ & 0.22 (0.21 to 0.24$)$ & 0.41 (0.40 to 0.42$)$ \\
\hline$>95$ & $0.26(0.22$ to 0.29$)$ & 0.39 (0.38 to 0.41$)$ & 0.27 (0.26 to 0.29$)$ & 0.44 (0.43 to 0.44$)$ \\
\hline Not stated & 0.01 & 0.02 & & \\
\hline
\end{tabular}

${ }^{*}$ Adjusted by capture-recapture.

1.48) (table 2). We conclude that prevalence has not decreased with time, but rather that age is the main determinant of prevalence.

To corroborate this conclusion, in September 2000 we reapproached six informants who maintain computerised records of large numbers of children. Two years previously they had notified 233 children in the cohorts 1992-5 inclusive. They now notified 336 children in the same cohorts. The increase is compatible with the hypothesis that confirmed prevalence rises with age.

\section{Discussion}

The prevalence of permanent childhood hearing impairment rises over a wider age range and to a higher plateau than has been reported previously. In our study data collection relied on notifications from professionals in health and education, not all of whom responded. However, geographical coverage was comprehensive, and the relation of our results to previously reported prevalence rates indicates that our methods of ascertainment were reliable.

Previous ascertainment studies included fewer children and a narrower range of cohorts and hence could not map the rise in prevalence across birth cohorts with the precision displayed in figure 3 . The profile of the rise in prevalence with age has important implications for service delivery, which can be dealt with only when the variables underpinning the rise are understood. Three effects are likely to contribute.

Some children acquire impairment postnatallyImpairments that are acquired, as distinct from progressive or of late onset, account for 4-9\% of overall prevalence $^{7}{ }^{15-16}$ and $7 \%$ in the present study. Thus they explain only a small proportion of the rise.

Confirmation of impairment is delayed in some children-Delayed confirmation of congenital cases may contribute to the rise, particularly for lesser degrees of impairment, ${ }^{7823}$ but if delay accounted for all of that part of the rise not attributed to acquired impairment, and if universal neonatal hearing screening identified all congenital impairments, then the yield from screening would be close to the aggregate prevalence in table 1. In fact, in the United Kingdom the yield of children with bilateral hearing impairment $\geqslant 40 \mathrm{~dB}$ HL per 1000 live births has been reported as only 1.18 $(34 / 28890)^{23}$ and $0.94(24 / 25609),{ }^{24}$ giving an aggregate yield of 1.06 (95\% confidence interval 0.84 to 1.44). This is close to the overall prevalence in the youngest cohort in our study, and the upper confidence limit is below the aggregate prevalence (table 1). Thus it is unlikely that delayed confirmation fully accounts for the unexplained portion of the rise.

Some inherited causes of hearing impairment manifest themselves only postnatally-Many of the dominant genes for deafness are associated with late onset progressive hearing impairment. ${ }^{25}{ }^{26}$ The protracted rise in the prevalence of severe and profound impairments (fig 3) is more compatible with the idea that some children have impairments of progressively increasing severity than with the alternative that many congenital cases with severe and profound impairments were not confirmed until several years after birth.

The implementation of universal neonatal hearing screening should result in a well documented

Table 2 Comparison of data from Trent ascertainment study ${ }^{7}$ and current study for children matched for birth cohort (same cohorts as Trent) and age range (same ages as Trent)

\begin{tabular}{|c|c|c|c|}
\hline & Trent ascertainment $1985-90$ & $\begin{array}{l}\text { Current study } 1985-90 \text { (same } \\
\text { cohorts as Trent) }\end{array}$ & $\begin{array}{c}\text { Current study } 1988-93 \text { (same } \\
\text { ages as Trent) }\end{array}$ \\
\hline Live births & 366480 & 4644400 & 4697700 \\
\hline \multicolumn{4}{|c|}{ Hearing level: overall ( $\geqslant 40 \mathrm{~dB}$ HL (Trent); $>40 \mathrm{~dB}$ HL (current)) } \\
\hline No of children & 487 & 7564 & 6773 \\
\hline Prevalence per 1000 live births $(95 \% \mathrm{Cl})$ & $1.33(1.22$ to 1.45$)$ & 1.63 (1.59 to 1.67$)$ & $1.44(1.41$ to 1.48$)$ \\
\hline Comparison of studies $\left(\chi^{2}\right)$ & - & 19.028; $P<0.001$ & $3.028 ; P=0.085$ \\
\hline \multicolumn{4}{|c|}{ Hearing level: moderate (40-70 dB HL (Trent); $41-70 \mathrm{~dB}$ HL (current)) } \\
\hline No of children & 270 & 4159 & 3739 \\
\hline Prevalence per 1000 live births $(95 \% \mathrm{Cl})$ & 0.74 (0.65 to 0.83$)$ & 0.90 (0.87 to 0.92$)$ & $0.80(0.77$ to 0.82$)$ \\
\hline Comparison of studies $\left(\chi^{2}\right)$ & - & 9.693; $P<0.01$ & $1.505 ; P=0.223$ \\
\hline \multicolumn{4}{|l|}{ Hearing level: severe (71-95 dB HL) } \\
\hline No of children & 104 & 1574 & 1379 \\
\hline Prevalence per 1000 live births $(95 \% \mathrm{Cl})$ & $0.28(0.23$ to 0.35$)$ & $0.34(0.32$ to 0.36$)$ & $0.29(0.28$ to 0.31$)$ \\
\hline Comparison of studies $\left(\chi^{2}\right)$ & - & $3.083 ; P=0.083$ & $0.111 ; P=0.764$ \\
\hline \multicolumn{4}{|l|}{ Hearing level: profound (>95 dB HL) } \\
\hline No of children & 113 & 1739 & 1580 \\
\hline Prevalence per 1000 live births $(95 \% \mathrm{Cl})$ & $0.31(0.26$ to 0.37$)$ & 0.38 (0.36 to 0.39$)$ & $0.34(0.32$ to 0.35$)$ \\
\hline Comparison of studies $\left(\chi^{2}\right)$ & - & $4.016 ; \mathrm{P}<0.05$ & $0.797 ; P=0.374$ \\
\hline
\end{tabular}




\section{What is already known on this topic}

The prevalence of confirmed permanent childhood hearing impairment ( $>40 \mathrm{~dB}$ HL) in the United Kingdom has been estimated to rise with age to $1.33 / 1000$ live births among children aged 5 years and older

It has been predicted that only an additional $16 \%$ of children will remain to be detected in the postnatal years, given current yields from universal neonatal hearing screening

\section{What this study adds}

The prevalence of confirmed permanent childhood hearing impairment ( $>40 \mathrm{~dB} \mathrm{HL})$ in the United Kingdom has risen with age to at least $1.65 / 1000$ live births (and may be as high as 2.05/1000 live births) among children 9 years of age and older

If the current yield from screening is sustained, then an additional $50-90 \%$ of children will remain to be detected in the postnatal years

screening history for all children. That information, together with the results of genetic investigations in children with a newly confirmed diagnosis of permanent hearing impairment, would permit the rising profile of prevalence with age to be confirmed prospectively and would allow us to unravel the relative contributions of the three effects to the rise.

\section{Conclusions}

In the United Kingdom the prevalence of confirmed cases of permanent childhood hearing impairment $>40 \mathrm{~dB}$ HL has risen with age to a significantly higher plateau than previous studies have estimated. Prevalence at the plateau is at least as high as the observed value of 1.65/1000 live births and may approach the adjusted value of $2.05 / 1000$ live births. The importance of these results rests on assumptions that prevalence at the plateau will not change in the foreseeable future and that the yield from universal neonatal hearing screening will remain close to $1.06 / 1000$ live births. If so, then for every 10 children with a permanent bilateral hearing impairment $>40$ $\mathrm{dB}$ HL detected by screening another five to nine children (50-90\%) would manifest such a hearing impairment by the age of 9 years. These additional children would comprise some with congenital impairments who either miss neonatal hearing screening or pass the screening despite having a hearing impairment, some who acquire an impairment postnatally, and others who manifest late onset or progressive impairments. Paediatric audiology and associated services will need the capacity and skills to identify and then confirm impairments in these children.
We thank every professional who identified children and Mrs Margaret Eatough for facilitating the ascertainment in the education sector.

Contributors: All authors contributed to the design and interpretation of the study and reviewed the manuscript Additionally HMF collected the data, DHM and AQS performed the analyses, and HMF and AQS drafted the manuscript. Danielle Betts and Sherie Holroyd assisted with the collection and entry of the data. HMF and AQS are guarantors.

Funding: Medical Research Council with additional funding from the National Lottery Charities Board through Defeating Deafness-The Hearing Research Trust.

Conflict of interest: None declared.

1 Conrad R. The deaf schoolchild: language and cognitive function. London: Harper and Row, 1979.

2 Wood D, Wood H, Griffiths A, Howarth I. Teaching and talking with deaf children. Chichester: Wiley, 1986.

3 Gregory S. Deaf children and their families. Cambridge: Cambridge University Press, 1995.

4 Cheng AK, Rubin HR, Powe NR, Mellon NK, Francis HW, Niparko JK. Cost-utility analysis of the cochlear implant in children. JAMA 2000;284:850-6.

5 Mohr PE, Feldman JJ, Dunbar JL, McConkey-Robbins A, Niparko JK, Rittenhouse RK, et al. The societal costs of severe to profound hearing loss in the United States. Int I Technol Assess 2000;16:1120-35.

6 Downs MP, Yoshinaga-Itano C. The efficacy of early identification and intervention for children with hearing impairment. Pediatr Clin N Am 1999;46:79-87

7 Fortnum H, Davis A. Epidemiology of permanent childhood hearing impairment in Trent region, 1985-1993. Br J Audiol 1997;31:409-46.

8 Van Naarden K, Decouflé P, Caldwell K. Prevalence and characteristics of children with serious hearing impairment in Metropolitan Atlanta. Pediatrics 1999;103:570-5

9 National Institutes of Health. NIH recommends universal screening of infants for hearing impairment. Am Fam Physician 1993;48:521-2.

10 Davis A, Bamford J, Wilson I, Ramkalawan T, Forshaw M, Wright S. A critical review of the role of neonatal hearing screening in the detection of congenital hearing impairment. Health Technol Assess 1997;1(10):1-176.

11 Dalzell L, Orlando M, MacDonald M, Berg A, Bradley M, Cacace A, et al The New York State universal newborn hearing screening demonstration project: ages of hearing loss identification, hearing aid fitting, and enrolment in early intervention. Ear Hearing 2000;21:118-30.

12 Department of Health. Piloting the introduction of universal neonatal hearing screening in England. www.doh.gov.uk/uhnspilots/index.htm (accessed 11 Jan 2001).

13 NHS Executive. Launch of pilot project to provide patients with digital hearing aids. www.doh.gov.uk/hearingaidproject/press0029.htm (accessed 11 Jan 2001).

14 Davis AC, Parving A. Towards appropriate epidemiological data on childhood hearing disability: a comparative European study of birth cohorts. J Audiol Med 1993;3:35-47.

15 Mäki-Torkko EM, Lindholm PK, Väyrynen MRH, Leisti JT, Sorri MJ. Epidemiology of moderate to profound childhood hearing impairments in Northern Finland. Any changes in ten years? Scand Audiol 1998;27: 95-103.

16 Uus K, Davis A. Epidemiology of permanent childhood hearing impairment in Estonia, 1985-1990. Audiology 2000;39:192-7.

17 LaPorte RE, McCarty DJ, Tull ES, Tajima N. Counting birds, bees, and NCDs. Lancet 1992;339:494-5.

18 International Working Group for Disease Monitoring and Forecasting. Capture-recapture and multiple-record systems estimation I: History and theoretical development. Am J Epidemiol 1995;142:1047-58.

19 Cormack RM. Problems with using capture-recapture in epidemiology: an example of a measles epidemic. J Clin Epidemiol 1999;52:909-14.

20 Bloor M, Wood F, Palmer S. Use of mark-recapture techniques to estimate the size of hard-to-reach populations. J Health Serv Res Policy 2000;5: 89-95.

21 Office for National Statistics. Population trends, summer 1999. London: Stationery Office, 1999

22 British Society of Audiology. Recommendations for pure-tone audiometry. Br J Audiol 1988;22:123.

23 Watkin PM, Baldwin M. Confirmation of deafness in infancy. Arch Dis Child 1999;81:380-9.

24 Kennedy CR. Controlled trial of universal neonatal screening for early identification of permanent childhood hearing impairment: coverage, positive predictive value, effect on mothers and incremental yield. Wessex Universal Neonatal Screening Trial Group. Acta Paediatr Suppl 1999;88:73-5.

25 Van Camp G, Smith RJH. Hereditary hearing loss homepage www.uia.ac.be/dnalab/hhh (accessed 11 Jan 2001).

26 Steel KP. New interventions in hearing impairment. BMJ 2000;320:622-5. (Accepted 25 May 2001) 


\title{
Commentary: Universal newborn hearing screening: implications for coordinating and developing services for deaf and hearing impaired children
}

\author{
Adrian Davis, Christine Yoshinaga-Itano, Sally Hind
}

Public Health and Clinical Section, MRC Institute of Hearing Research, University Park,

Nottingham NG7 2RD

Adrian Davis head of epidemiology, public health and clinical sections

Sally Hind

developmental

psychologist

Department of Speech, Language, and Hearing Sciences, University of Colorado, Boulder 80309-0409, Colorado, USA Christine Yoshinaga-Itano professor

Correspondence to: A Davis Adrian@ihr. mrc.ac.uk
The prevalence of permanent childhood hearing impairment of $40 \mathrm{~dB}$ HL or greater, and the probability that late onset and progressive hearing impairment may be more prevalent than previously indicated, has been discussed by Fortnum et al in their paper. There is no estimate available of the number of children with "mild" (20-40 dB HL) bilateral permanent impairment or those with unilateral impairment, but current programmes that screen for hearing problems in the newborn in the United States suggest that such impairment, identified by screening at birth, is at least as prevalent.

Screening hearing in newborns has been shown to be efficient ${ }^{2}$ and cost effective, ${ }^{3}$ with a sensitivity in the range of $80-90 \%$, a false positive rate of $<2 \%{ }^{45}$ and a positive predictive value of $17 \%$. The proposed costs of such screening in the United Kingdom are much lower than the costs of the current infant distraction screen test, ${ }^{8}$ and the cost per child identified as having bilateral permanent hearing impairment is considerably less. There is little evidence that screening all newborns for hearing raises anxiety among mothers. ${ }^{6}$

The benefits of universal newborn hearing screening for children with permanent hearing impairment are that early identification is associated with better expressive and receptive language, speech, and social and emotional development. Children who are identified before the age of 6 months show substantial benefit in the first five years of life, and there is some evidence that earlier enrolment in intervention programmes is associated with better outcomes. ${ }^{7}$ A higher level of expressive language in young children is linked with levels of parental stress and better attachment as measured by emotional availability. ${ }^{89}$ However, if early identification and intervention is not handled well at the service level, it can generate anxiety and grief and bring about negative outcomes for the family. ${ }^{10}$

The Department of Health has started a universal newborn hearing screening programme in England, beginning with a pilot implementation with a hospital or clinic based protocol in 17 areas and a community based protocol in three areas. Concerns over the quality of services for the assessment of children's hearing for those referred from screening and of early intervention programmes for parents and their children who have a confirmed permanent hearing impairment have been raised. Family Friendly Hearing Services are being developed that have three main characteristics. Firstly, service provision by all professional sectors in a positive family friendly culture should encourage "seamless" collaboration, responsiveness that meets the family's real needs, and provision of appropriate information between all agencies and for parents that enables families to make informed choices about services for their children. Sec- ondly, paediatric audiology should exceed a minimum standard in terms of quality and accessibility. Thirdly, there must be a culture of service evaluation, including peer review, with an element of feedback from parents and their children with impaired hearing. Data from screening and assessments will be kept nationally on a database integrated with other child services. This will facilitate the monitoring of later development of permanent childhood hearing impairment and the effectiveness of the screening programme.

The implementation team will rigorously assess access to and quality of health and social care for these families, and outcomes of the programme will be evaluated. Coordination of these services and support options are key factors in the success of the programme, and availability of the range of options would be severely restricted by a lack of appropriately trained staff and resources. The aim is to detect bilateral moderate to profound congenital permanent childhood hearing impairment to enable high quality parent-child intervention services.

The advent of a national newborn hearing screening programme creates the opportunity to help these children to develop their true potential, provided that the training, resources, and coordination are made available. We will need to monitor the outcomes of the children at different ages to enhance the evidence base concerning the most effective health, educational, and social interventions. There continues to be a need to develop and implement more effective screening and case finding for school aged children (4-16 years) with acquired and late onset hearing impairments that may negatively affect their behaviour and educational achievement.

1 Dalzell L, Orlando M, MacDonald M, Berg A, Bradley M, Cacace A, et al. The New York State universal newborn hearing screening demonstration project: ages of hearing loss identification, hearing aid fitting, and enrolment in early intervention. Ear Hear 2000;21:118-30.

2 Davis A, Bamford J, Stevens J. Performance of neonatal and infant hearing screens: sensitivity and specificity. Br J Audiol 2001;35:3-15.

3 Stevens JC, Hall DM, Davis A, Davies CM, Dixon S. The costs of early hearing screening in England and Wales. Arch Dis Child 1998;78:14-9.

4 Yoshinaga-Itano C, Coulter D, Thomson V. The Colorado newborn hearing screening project: effects on speech and language development for children with hearing loss. J Perinatol 2000;20:S132-7.

5 Kennedy C, Kimm L, Thornton R, Davis A. False positives in universal neonatal screening for permanent childhood hearing impairment. Lancet 2000;356:1903-4.

6 Watkin PM, Baldwin M, Dixon R, Beckman A. Maternal anxiety and attitudes to universal neonatal hearing screening. Br J Audiol 2000;32:27-37. Moeller MP. Early intervention and language development in children who are deaf and hard of hearing. Pediatrics 2000;106:E43.

8 Pressman L, Pipp-Siegel S, Yoshinaga-Itano C, Deas A. Maternal sensitivity predicts language gain in preschool children who are deaf and hardof-hearing. J Deaf Studies Deaf Education 1999;4:294-304.

9 Pressman L, Pipp-Siegel S, Yoshinaga-Itano C, Kubicek L, Emde RN. A comparison of the links between emotional availability and language gain in young children with and without hearing loss. Volta Review 2000;100:251-77.

10 Hind S, Davis A. Outcomes for children with permanent hearing impairment. In: Seewald R, ed. A sound foundation through early amplification. Proceedings of the International Conference sponsored by Phonak Oct 1998, Chicago, Illinois, US. Staefa, Staefa, Switzerland: Phonak AG, 2000:199212. 